\title{
Ureteral endometriosis following panhysterectomy a case report
}

\section{Sendhil Coumary Arumugam ${ }^{1} *$ Kavitha Senthil $^{2}$, Hemalatha Pugalendhi ${ }^{3}$}

\author{
${ }^{1}$ Department of Obstetrics and Gynecology, Mahatma Gandhi Medical College \& Research Institute, Pillaiyarkuppam \\ Puducherry, 605006, India \\ ${ }^{2}$ Pankajam Sitharam Nursing Home Srirangam, and GVN hospitals Trichy, India \\ ${ }^{3} \mathrm{GVN}$ hospitals Trichy, India
}

Received: 22 September 2015

Accepted: 01 November 2015

\section{*Correspondence:}

Dr. Sendhil Coumary Arumugam,

E-mail: sendhilcoumary1975@yahoo.in

Copyright: ( ) the author(s), publisher and licensee Medip Academy. This is an open-access article distributed under the terms of the Creative Commons Attribution Non-Commercial License, which permits unrestricted non-commercial use, distribution, and reproduction in any medium, provided the original work is properly cited.

\begin{abstract}
Ureteral endometriosis is a rare form of endometriotic lesion. It can be intrinsic or extrinsic, intrinsic being more rare than extrinsic. Extrinsic ureteral involvement can lead to silent damage to the kidneys. It is commonly seen in women with rectovaginal endometriosis. They present with a variety of symptoms from flank pain to hematuria. Though it responds to medical management, it will require definitive surgical intervention such as a simple cyst excision to the extent of ureteric resection and reanastamosis.
\end{abstract}

Keywords: Endometriosis, Ureter, Post menopausal.

\section{INTRODUCTION}

Ureteral endometriosis is relatively uncommon and is estimated to occur in less than $0.3 \%$ of all endometriotic lesions. Around 300 cases of ureteral endometriosis have been reported since 1996. Ureteral involvement may lead to silent loss of kidney function. There is an increasing incidence of ureteral involvement attributable to better imaging modalities. ${ }^{1} 4.4 \%$ of women suffering from rectovaginal adenomyosis have ureteric involvement. ${ }^{2}$ In those patients with ureteric obstruction as many as $25 \%$ will sustain irreversible renal damage because of the delay in diagnosis. The frequency of endometriosis is rare among postmenopausal women; around $2.2 \%$. $^{3}$ Here we report a rare case of ureteral endometriosis in a woman who had attained surgical menopause.

\section{CASE REPORT}

Mrs. M, a 35 year old P2 L2 presented to the OPD with fever, chills, rigors and lower abdomen pain of ten days duration. She had undergone abdominal hysterectomy with bilateral salpingo-oophorectomy seven years ago in view of adenomyosis of the uterus with bilateral ovarian endometriotic cysts. She had received three doses of leuprolide following surgery. She had also received some form of HRT for one year which she later discontinued on her own.

On examination she was febrile with tachypnoea and tachycardia. Abdomen examination revealed severe left loin tenderness. Urgent urine examination revealed presence of infection and urine culture yielded heavy growth of Escherichia coli.

USG of the abdomen revealed a thick walled cyst with low level internal echoes measuring $3 \times 3 \mathrm{~cm}$ in the left side of pelvis suggestive of endometriotic cyst. The cyst was seen compressing left lower ureter and causing hydroureteronephrosis (Figure 1) and an intravenous pyelogram revealed the same (Figure 2). A contrast CT of the abdomen confirmed the finding (Figure 3). DMSA study revealed diminished left renal function (Figure 4). A diagnosis of endometriotic cyst causing left hydroureteronephrosis presenting as acute pyelonephritis was made. 


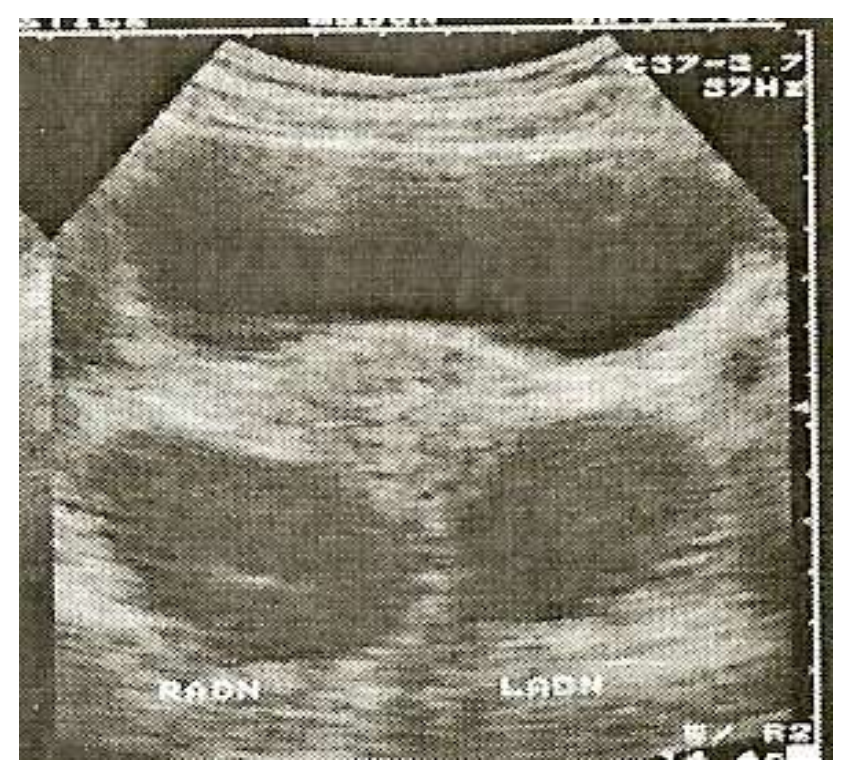

Figure 1: Ultrasound showing loculated collection in left adnexa suggestive of endometriotic cyst.

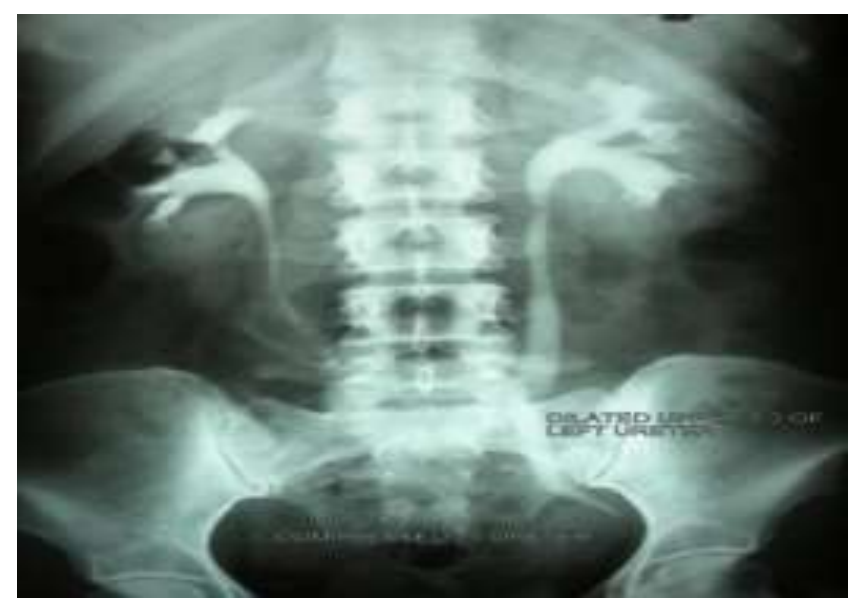

Figure 2: IVP showing dilated left ureter.

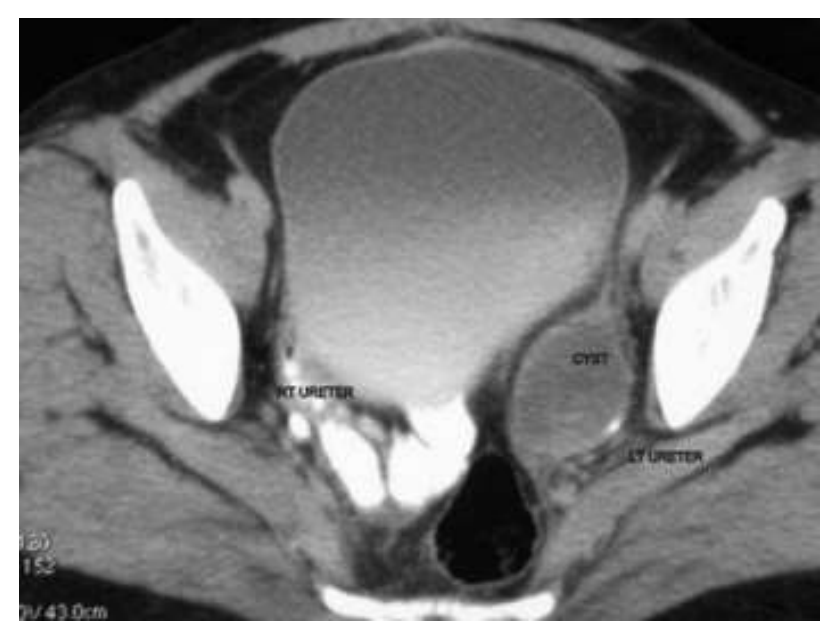

Figure 3: CT scan showing left ovarian endometrioma.

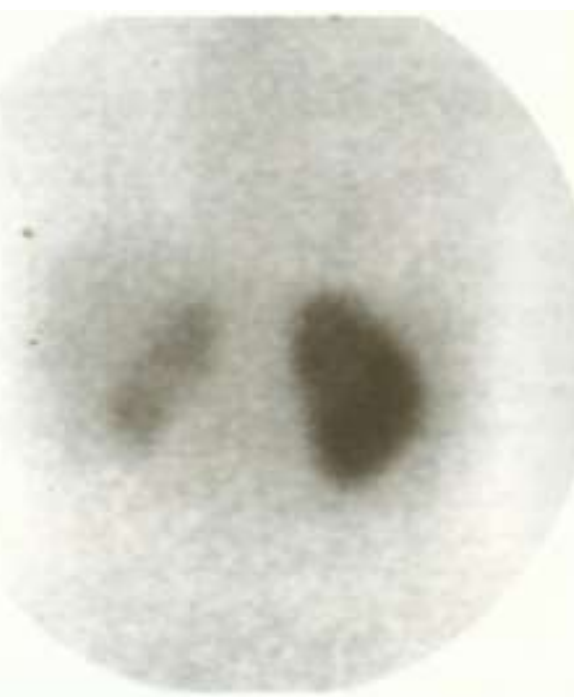

Figure 4: DMSA showing delayed excretion of dye from the left kidne.

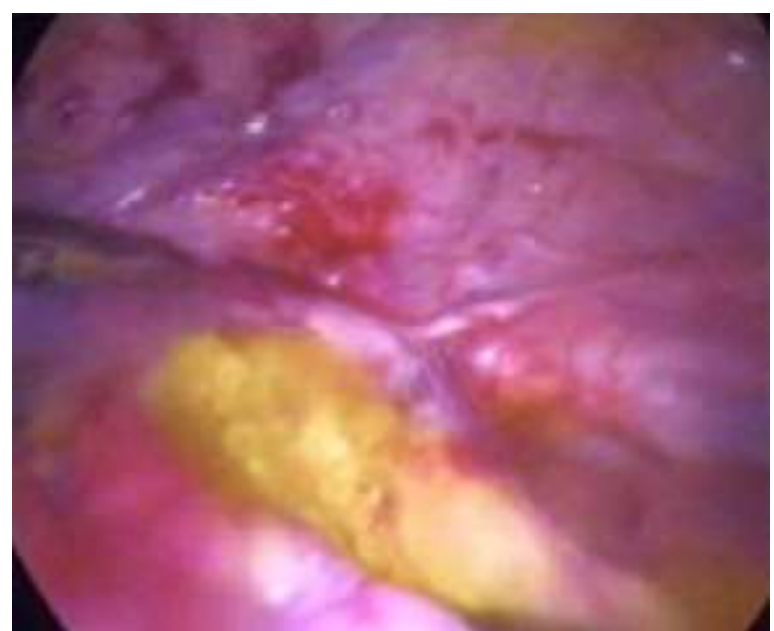

Figure 5: Laproscopic picture showing left endometriotic cyst.

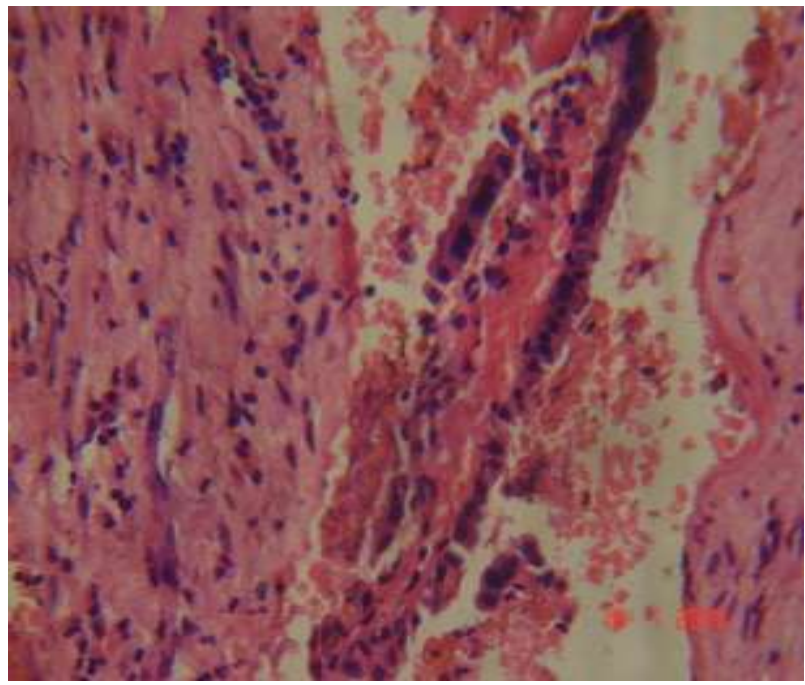

Figure 6: Endometrial gland in the excised nodule of the ureteral endometrioma. 
Under antibiotic cover ureteral stenting was initially done. Once stable, patient underwent laproscopic ureterolysis with excision of endometriotic cyst (Figure 5). Post operative period was uneventful and the ureteric stent removed after three months. She is currently on follow up with no further recurrence. Histopathology revealed presence of endometrial glands and stroma in the tissues adjacent to the ureter (Figure 6).

\section{DISCUSSION}

Ureteral endometriosis occurs in 0.1-1\% of women with endometriosis, with a peak age incidence of 30-35 years. The mean age was 36.2 years in a study of 23 cases by $\mathrm{Mu}$ et al. It is usually rare in post menopausal women except those women who have been on prolonged estrogen supplementation. ${ }^{1,4}$

It is more common in the left lower third of the ureter. The predilection to the left can be explained by menstrual reflux theory and the anatomy of left hemipelvis making it more susceptible in the left as seen in ovarian endometriosis. In 10-20\% of cases it is bilateral. Ovarian endometriosis is a pre requisite for ureteral endometriosis. ${ }^{1}$

The two types of ureteral endometriosis are extrinsic and intrinsic ureteral endometriosis with the ratio being $4: 1$. Extrinsic ureteral endometriosis occurs within the ureteral adventitia and adjacent soft tissues only as seen in the present case. The extrinsic type of endometriosis usually results in external compression of the pelvic ureter. ${ }^{5}$

Intrinsic ureteral endometriosis is characterized by the presence of endometriotic glands and stroma within the muscularis propria, lamina propria or ureteral lumen due to primary involvement of ureteral wall. It is a very rare entity. ${ }^{1,5}$

Symptoms associated with ureteral endometriosis are varied. Flank pain, back ache, abdominal pain, urgency, frequency, haematuria etc. Since it is frequently associated with pelvic and rectovaginal endometriosis, symptoms related to them like dysmenorrhea, dyspareunia, chronic pelvic pain, constipation, diarrhea, dyschezia, tenesmus, hematochezia, infertility can also be associated. ${ }^{1,6}$

Histological study of periureteral resection specimens revealed the presence of endometrial glands in contact with the uterine artery and the ureter. Periarterial invasion

by the retroperitoneal adenomyotic disease was demonstrated histologically in all cases where, the uterine artery was resected. Similarly the point of ureteric obstruction was noted to occur most commonly at a small segment of distal left ureter, where it is crossed by the uterine artery. As endometriosis is a cicatrizing disease the disease process often seems to follow the course of the adjacent blood vessels such as uterine artery.
Immunostains for CK7 and PR was positive in all cases, whereas ER and CA125 was positive in $83 \%$ and $67 \%$ of the cases respectively. ${ }^{7}$

In resected specimens of ureter with ureteral endometriosis the deposits showed features of adenomyosis (ie) hyperplasia of smooth muscle cells along scant endometriotic glands and stroma. It was also suggested that the ureteric compression results from endometriosis of the retroperitoneal space probably from remanants of the mullerian duct. An incidence of $0.9 \%$ of ureteral endometriosis has been estimated in women with rectovaginal adenomyosis especially when the nodules were more than $3 \mathrm{~cm}$ in size. ${ }^{1}$ Concomitant endometriotic sites with ureteral endometriosis were ovarian (69.2\%), bowel endometriosis (61.5\%), uterosacral ligaments $(69.2 \%)$ and obliterated cul de sac $(38.5 \%)$ in a series of 126 women who underwent ureterolysis for ureteral endometriosis.

The ultrasound features of endometriomas are quite variable and sometimes mimic other benign and malignant ovarian lesions. Homogenous, hypoechoic cystic, with diffuse lower level internal echoes, within the ovary is suggestive of ovarian endometriosis. Ultrasonography is the most appropriate noninvasive, initial investigation for assessing ureteric obstruction. The presence of hydronephrosis in ultrasound along with an ovarian endometriotic lesion should raise the suspicion of ureteral endometriosis. In a study of 23 cases of ureteric endometiosis by $\mathrm{Mu}$ et al, all 23 patients showed evidence of ureteric involvement on ultrasound. ${ }^{6}$ Almost all imaging techniques are of limited value in providing an accurate depiction of the extent of the disease and the infiltration of the ureteral wall. IVU has been of traditional use in demonstrating the precise location, extent and degree of ureteral stenosis as well as to confirm renal function, though the current gold standard would be MRI especially along with MR urography. ${ }^{1,8}$

Imaging is very important in endometriosis to plan management, patient counseling and also to anticipate problems during surgery. MRI represents the ideal 'all-inone' imaging in patients with suspected ureteral endometriosis, because it allows the visualization of all components of the urinary system and the possibility of exploring all pelvic locations of endometriosis. ${ }^{1}$ The implants demonstrate low signal intensity on T2 weighted MR images and high signal on T1 images. Ureteroscopy has been used to diagnose intrinsic type of ureteral endometriosis. This examination allows for both direct observations of the bladder and ureteral lesions and providing biopsy specimens for histology. A negative scopy does not rule out extrinsic ureteral endometriosis. ${ }^{1}$

If rectovaginal endometriosis is diagnosed on physical examination especially if more than $3 \mathrm{cms}$, sonography of the kidney, an intravenous pyelography or MRI is of use to diagnose or exclude ureteral obstruction. If ureteral 
obstruction is diagnosed renography is indicated to diagnose loss of kidney function.

The three main aims in the management of ureteral endometriosis are relief of obstruction, preserve renal function and avoid relapse of disease. It may be medical surgical or both. ${ }^{9}$

Medical management in the form of hormonal manipulation will help regression of lesions though the fibrosis from residual disease may compromise renal function. It should be used as an adjunct to surgical management. Locally released progestrones like levonorgestrol, GnRH analogues and combined pills can be used. ${ }^{9}$ Patient may be symptomatically relieved, though the symptoms may recur with stoppage of medical treatment especially with deep infilterating endometriosis.

Surgical treatment is especially indicated in the presence of hydroureteronephrosis. The use of open or minimally invasive techniques remains controversial. It may range from ureterolysis, segmental resection and anastomoses or ureteroneocystostomy. Pre operative ureteric stenting is however a must. Also, the choice surgery depends on the type of ureteric lesion, whether intrinsic or extrinsic. Despite surgery, a few may have residual compromised kidney function. A combined effort from the gynecologist, radiologist and the urologist is required to salvage the remaining renal function in a case of ureteral endometriosis.

Currently robot assisted minimally invasive procedures form yet another option for ureterolysis and ureteric resection reanastomosis. ${ }^{10}$ Firefly $^{\mathrm{TM}}$ technology and indocyanine green facilitate identification of endometriosis in single-site robotic surgery, as the endometriotic deposits are associated with a high degree of neovascularization. This enables them to perform single-site laparoscopic resection of advanced endometriotic nodules overlying the ureter and rectum with complete resolution of pelvic pain symptoms and excellent cosmetic results. ${ }^{11}$

Women with endometriotic cysts and associated hydroureteronephrosis of the same side must have further investigations to rule out ureteric involvement. This must be irrespective of their age; because with increasing use of harmone replacement therapy there seems to be an increasing evidence of endometriosis even among postmenopausal women. ${ }^{3,4}$ It is important to be aware of this, to avoid silent loss of kidney function.
Funding: No funding sources

Conflict of interest: None declared

Ethical approval: Not Required

\section{REFERENCES}

1. Maccagnano C, Pellucchi F, Rocchini L, Ghezzi M, Scattoni V, Montorsi F, Rigatti P, Colombo R. Ureteral Endometriosis: Proposal for a Diagnostic and Therapeutic Algorithm with a Review of the Literature Urol Int. 2013;91:1-9.

2. Donnez J, Nisolle $M$, Squifflet J. Ureteral endometriosis: a complication of rectovaginal endometriotic (adenomyotic) nodules. Fertil Steril. 2002;77(1):32-7.

3. Punnonen R, Klemi PJ, Nikkanen V. Postmenopausal endometriosis. Eur J Obstet Gynecol Reprod Biol. 1980;11(3):195-200.

4. Oxholm D, Knudsen UB, Kryger-Baggesen N, Ravn P: Postmenopausal endometriosis. Acta Obstet Gynecol Scand 2007,86:1158-64.

5. Koninckx PR, Ussia A, Adamyan L, Wattiez A, Donnez J. Deep endometriosis: definition, diagnosis, and treatment. Fertil Steril. 2012;98(3):564-71.

6. Al-Khawaja M, Tan PH, MacLennan GT, LopezBeltran A, Montironi R, Cheng L. Ureteral endometriosis: clinicopathological and immunohistochemical study of 7 cases. Hum Pathol. 2008;39(6):954-9.

7. Dawei Mu, Xuesong Li, Zhou G, Guo H. Diagnosis and Treatment of Ureteral Endometriosis: Study Of 23 Cases Urology Journal. 2014;1806-12.

8. Paula J. Woodward, Sohaey R, Mezzetti TP. LCDR, USNR, MC Endometriosis: Radiologic-Pathologic Correlation From the Archives of the AFIP

9. Ureteral endometriosis treated by hormonal therapy: a case report Mikami J, Yamamoto $\mathrm{H}$, Okamoto A, Ishimura $\mathrm{H}$, Imai $\mathrm{A}$, Hatakeyama $\mathrm{S}$, Yoneyama $\mathrm{T}$, Hashimoto Y, Koie T, Ohyama C. Hinyokika Kiyo. $2013 ; 59(7): 431-4$.

10. Frick AC, Barakat EE, Stein RJ, Mora M, and Falcone T. Robotic-Assisted Laparoscopic Management of Ureteral Endometriosis JSLS. 2011; 15(3):396-9.

11. Guan X, Nguyen MT, Walsh TM, Kelly B. Robotic Single Site Endometriosis Resection Using Firefly Technology J Minim Invasive Gynecol. 2015;5534650(15)00608-1.

Cite this article as: Arumugam SC, Senthil K, Pugalendhi H. Ureteral endometriosis following panhysterectomy a case report: a rare case report. Int J Reprod Contracept Obstet Gynecol 2015;4:207780. 